\title{
Antigen-specific regulatory T-cell responses to intestinal microbiota
}

\author{
EV Russler-Germain ${ }^{1}$, S Rengarajan ${ }^{1}$ and C-S Hsieh ${ }^{1}$
}

The mammalian gastrointestinal tract can harbor both beneficial commensal bacteria important for host health, but also pathogenic bacteria capable of intestinal damage. It is therefore important that the host immune system mount the appropriate immune response to these divergent groups of bacteria-promoting tolerance in response to commensal bacteria and sterilizing immunity in response to pathogenic bacteria. Failure to induce tolerance to commensal bacteria may underlie immune-mediated diseases such as human inflammatory bowel disease. At homeostasis, regulatory $\mathrm{T}$ (Treg) cells are a key component of the tolerogenic response by adaptive immunity. This review examines the mechanisms by which intestinal bacteria influence colonic T-cells and B-cell immunoglobulin A (IgA) induction, with an emphasis on Treg cells and the role of antigen-specificity in these processes. In addition to discussing key primary literature, this review highlights current controversies and important future directions.

\section{INTRODUCTION}

The mammalian gastrointestinal tract is adapted to harbor trillions of bacteria, ${ }^{1}$ many of which provide beneficial functions to the host, including protection from infection by pathogenic organisms ${ }^{2,3}$ and nutrient metabolism., However, the close physical proximity of these bacteria to the host represents a unique challenge to the immune system, as it must be able to discriminate pathogenic bacteria from those normally resident in the gut. Inappropriate tolerance to pathogens may facilitate infection, ${ }^{6,7}$ whereas immune reactivity against harmless commensal microbiota is thought to underlie the pathogenesis of human inflammatory bowel disease $^{8,9}$ (IBD). Thus, the generation of appropriate immune responses to bacteria is crucial for intestinal health.

Although intestinal homeostasis requires multiple arms of the immune system, ${ }^{10}$ here we will focus on the role of T-cell responses to commensal bacteria. The absence of adaptive immune T-cells leads to a failure of gut homeostasis in murine models, with bacterial translocation and colitis. ${ }^{11}$ Similarly, intestinal pathology without obvious pathogenic infection also happens in humans with HIV that is associated with decreased CD4 ${ }^{+}$T-cell counts. ${ }^{12,13}$ Adaptive immune cells are therefore not only required to target pathogenic bacteria, ${ }^{14,15}$ but also recognize and control normal intestinal bacteria during homeostasis.
However, the predominant $\mathrm{T}$-cell responses to intestinal bacteria during homeostasis are likely inhibitory responses to limit inflammation and immune-mediated gut pathology, rather than effector responses to eliminate bacteria. It is now generally accepted that tolerance to intestinal bacteria requires $\mathrm{CD}^{+}{ }^{+}$regulatory $\mathrm{T}$ (Treg) cells, as first suggested in adoptive transfer experiments in rodents. ${ }^{16}$ Treg cells are defined by the transcription factor Foxp3, which is required for Treg cell function and development. ${ }^{17}$ In humans, patients with Treg cell deficiency (IPEX; immune dysregulation, polyendocrinopathy, enteropathy, X-linked) exhibit diarrhea and at times colitis among other autoimmune disease manifestations. ${ }^{17}$ Thus, Treg cell-mediated tolerance to gut bacteria is crucial for maintenance of immune homeostasis and prevention of IBD.

We will review our current understanding of the reciprocal interactions of T-cells and intestinal bacteria during homeostasis. Specifically, we will discuss the roles of intestinal bacteria in shaping tolerogenic Treg cell responses via nonantigen-specific factors, as well as address how antigenspecificity appears to be important for intestinal Treg cell development and function. Although other immunoregulatory T-cell subsets will not be addressed in this review, it is important to note that they make important contributions to gut tolerance. ${ }^{18-21}$ We will also explore the function of Treg and

${ }^{1}$ Department of Internal Medicine, Division of Rheumatology, Washington University School of Medicine, St. Louis, Missouri, USA. Correspondence: C-S Hsieh (chsieh@wustl.edu) 
effector T-cells during homeostasis and their roles in modulating antigen-specific immunoglobulin $\mathrm{A}$ (IgA) induction. As bacteria-dependent immunopathology in the small intestine is less common in humans, we will focus on Treg cell: bacteria interactions in the colon. Throughout, we will discuss discrepancies in conclusions formulated from primary data as well as identify unanswered questions in the field.

\section{DEVELOPMENTAL ORIGIN OF COLONIC TREG CELLS}

It is now well-established that the colonic Treg cell population is affected by intestinal bacteria. ${ }^{10}$ For example, germ-free mice show a several-fold reduction in the frequency of Treg cells compared with conventionally housed specific pathogen free (SPF) mice. ${ }^{22,23}$ This process does not require a complex microbiota, as introduction of individual bacterial isolates or defined consortia into germ-free mice is sufficient to induce colonic Treg cells. ${ }^{22,23}$

One mechanism by which intestinal bacteria can influence Treg cell numbers is by inducing expansion of pre-existing thymic Treg (tTreg) cells. Classically, tTreg cells are generated in response to antigen recognition at an immature stage of $\mathrm{T}$-cell development before their release into the periphery. ${ }^{24}$ Although it may be possible that colonic bacterial antigens are transported and presented in the thymus to induce tTreg cells, there is currently no evidence that this occurs. A more compelling possibility is that some self-antigen reactive tTreg cells exhibit cross-reactivity with foreign antigens, ${ }^{25}$ resulting in expansion of those bacterial-reactive clones in the intestines. Consistent with these possibilities, a marked overlap between the tTreg and colonic Treg TCR repertoires was observed in one study. ${ }^{26}$

Another mechanism is that intestinal bacteria can induce the peripheral differentiation of Treg ( $\mathrm{pTreg}$ ) cells from naive $\mathrm{T}$-cells. There are several lines of data suggesting that pTreg cells comprise the majority of the colonic Treg population. First, the use of markers reported to identify tTreg versus pTreg cells suggest that gut bacteria induce pTreg cells. High expression of the transcription factor Helios and cell surface protein Nrp-1 have been associated with tTreg cells. ${ }^{27-29}$ Although the utility of these markers remains controversial, ${ }^{30-32}$ colon lamina propria (cLP) Treg cells contain a much lower proportion of $\mathrm{pTreg}$ cells in germ-free compared with SPF mice based on these markers. ${ }^{22,27-29,33}$ In addition, treatment of SPF mice with broad-spectrum antibiotics results in the loss of cLP pTreg cells based on Helios levels. ${ }^{22}$ Second, deletion of the conserved noncoding sequence 1 (CNS1) in the Foxp3 locus results in a marked decrease in $\mathrm{p}$ Treg, but not tTreg, cell induction. $^{34,35}$ Notably, CNS1-deficient mice show fewer intestinal Treg cells at the time of weaning. ${ }^{34,35}$ Of the remaining intestinal Treg cells, there is a lower proportion of Nrp-1 $1^{\text {lo }}$ cells, consistent with loss of pTreg cells in CNS1deficient mice. ${ }^{28}$ Third, our analysis of colonic Treg TCRs showed that they were unable to induce tTreg cell selection. ${ }^{36}$ The different conclusion from the aforementioned TCR repertoire analyses ${ }^{26}$ may relate to the different analytical approaches used to assess tTreg cell selection-intrathymic injection of TCR-expressing thymocytes ${ }^{36}$ vs. comparison with thymic TCR repertoires. ${ }^{26}$ The conclusions may also be affected by the different TCR $\beta$ chains used ${ }^{26,36}$ or the use of a limited TCR $\alpha /$ fixed TCR $\beta$ model, ${ }^{26}$ as effects on Treg cell selection have been reported for changes in a single TCR chain (TCR $\alpha$ [ref. 37]). The ability of two of these colonic TCRs (CT2/CT6) to facilitate pTreg cell induction from naive T-cells in the periphery in normal mice was confirmed via the generation of transgenic (Tg) lines. ${ }^{33}$ Altogether, these data suggest that the majority of colonic Treg cells arise via pTreg cell differentiation from naive T-cells.

pTreg cells specific for intestinal bacteria appear to be important for colonic homeostasis. For example, treatment of experimental colitis with Treg cells was more effective using cotransfer of normal Treg cells plus naive Foxp $3^{-}$conventional T-cells (Tconv) cells that became pTreg cells, than by cotransfer of Treg cells alone. ${ }^{38}$ In conjunction with TCR repertoire analysis, it was suggested that the induced pTreg cells prevented colitis by providing additional TCR specificities not present in the transferred Treg cell population, which were mostly tTreg in origin. ${ }^{39}$ Similarly, mice that have decreased induction of $\mathrm{p}$ Treg, but not $\mathrm{t}$ Treg, cells through genetic deletion of the CNS1 Foxp3 enhancer region, eventually develop spontaneous colitis characterized by plasmacytic enteritis and high $\mathrm{CD} 4{ }^{+} \mathrm{T}$ cell expression of Th2 cytokines. ${ }^{34}$ As in other tissues, tTreg cells specific for self-antigens such as those in intestinal epithelial cells are also involved in maintaining homeostasis in the gut, ${ }^{16,22,40}$ and may expand specifically in the context of certain types of intestinal perturbations such as sterile injury. ${ }^{41}$ However, the above data suggest that $\mathrm{tTreg}$ cells are insufficient to maintain intestinal tolerance and that pTreg cells with a unique set of bacterial antigen specificities are required for homeostasis.

Our group has used colon Treg TCR Tg lines to study the process of pTreg cell differentiation to commensal bacterial antigens. ${ }^{33}$ Using adoptive transfer of peripheral naive T-cells from CT2/CT6 TCR Tg mice into wild type, lymphoreplete mice, it was found that $\mathrm{pTreg}$ cell generation was very efficient and resulted in over $80 \%$ of cells in the colon and mesenteric lymph nodes upregulating Foxp3 by three weeks after transfer. This was associated with induction of Foxp3 in the most proliferated $\mathrm{T}$ cell population, and is in stark contrast with previous studies in non-mucosal tissues showing $\mathrm{pTreg}$ cells in the least-divided population. ${ }^{42,43}$ These colonic TCR Tg data therefore demonstrate that $\mathrm{pTreg}$ cell generation can be the dominant outcome of naive T-cell activation.

TCR Tg cells from these two lines did not undergo Treg cell induction upon transfer in young mice, but only in mice around the age of weaning or thereafter. ${ }^{33}$ This was due to the lack of the appropriate bacteria in young mice, rather than an immature immune compartment incapable of inducing Treg cell selection, as fecal microbiota transplant from three-, but not one-, week old donors into one-week old recipients was sufficient to induce Treg cell development. The time-period around weaning is associated with major changes in the composition of the intestinal microbiota due to the switch to solid food and acquisition of a more adult-like microbiota. ${ }^{44}$ In fact, studies 


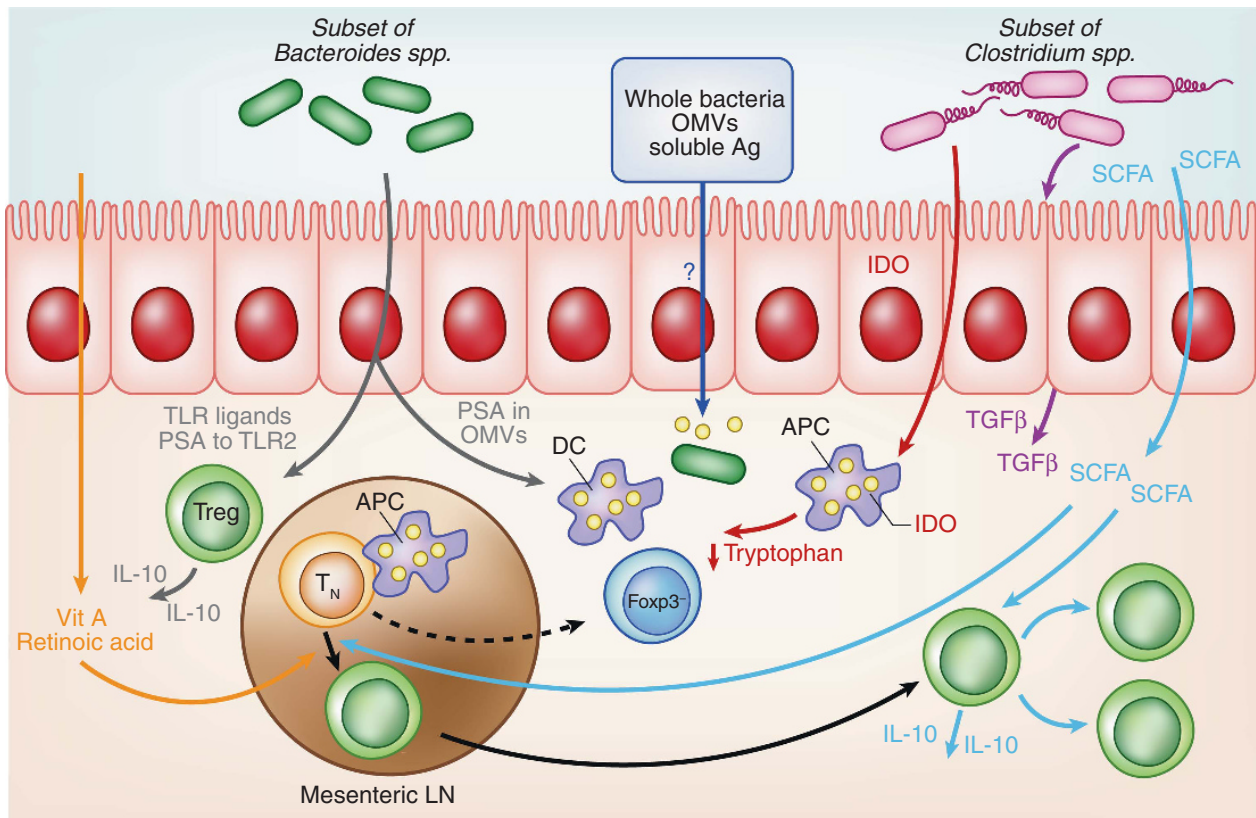

Figure 1 Antigen-specific and non-specific mechanisms of bacteria-induced treg cell generation and function. Clostridium spp. establish an environment favorable for $p$ Treg cell generation via the induction of TGF $\beta$ secretion by intestinal epithelial cells (IECs) (purple arrow), indoleamine-2,3dioxygenase in IECs and APCs (red arrows), and the production of SCFAs from dietary components (light blue). Bacteroides spp. such as B. fragilis have been described to increase Treg cell function through PSA activation of TLR2 ligation on T-cells and DCs (gray arrows). PSA, as well as SCFAs (blue arrow), induce IL-10 secretion from LP Treg cells. SCFAs also act directly on T-cells to induce Treg differentiation/expansion. Dietary vitamin A from the intestinal lumen (orange arrows) can enhance pTreg cell differentiation through its metabolite retinoic acid, which can affect pTreg cell selection by inducing transcription factor binding to a Foxp3 enhancer element in CNS1, ${ }^{48-50}$ or via blockade of effector cytokine production by effector T-cells. ${ }^{129}$ Bacterial antigens (dark blue arrow), may gain access to immune cells by a variety of mechanisms that are currently unclear, including via gobletassociated passages, transcytosis through IECs, extension of APC processes into the intestinal lumen, or direct invasion of whole bacteria. ${ }^{130,131}$ Naive T-cells have been reported to encounter commensal antigens in the mesenteric lymph node and undergo pTreg cell selection. ${ }^{33}$ The Peyer's patches and isolated lymphoid follicles may also be important sites of pTreg cell selection, but this requires further study.

using Helios and Nrp-1 to identify Treg cell origin show a marked shift in the colon from tTreg to pTreg cells around weaning. ${ }^{22,23,27,33,36}$ In summary, there is now a substantial body of work supporting the notion that the majority of colonic Treg cells are generated by pTreg cell selection in response to colonic bacteria acquired around weaning.

\section{MECHANISMS OF COLONIC PTREG CELL GENERATION}

The molecular mechanisms involved in colon pTreg cell development have been recently reviewed ${ }^{45}$ which we will briefly summarize (Figure 1). TGF $\beta$, an important factor for the generation of pTreg cells, ${ }^{46}$ can be induced by bacteria such as Clostridium from epithelial cells. ${ }^{47}$ Retinoic acid, a metabolite of dietary vitamin $\mathrm{A}$, has been shown to augment the Foxp3-inducing effects of TGF $\beta$ in vitro. ${ }^{48-50}$ Indoleamine2,3-dioxygenase can be produced by dendritic cells and intestinal epithelial cells in response to gut bacteria to favor pTreg cell differentiation. ${ }^{51,52}$ Short chain fatty acids (SCFAs) produced by gut bacteria from dietary components increase the percentage of Treg cells in the $\mathrm{CD} 4^{+} \mathrm{T}$ cell subset as well as the expression of IL-10 in Treg cells. ${ }^{53-56}$ SCFAs can inhibit histone deacetylase and via epigenetic modification confer greater stability to Foxp3 gene expression and increase pTreg differentiation. ${ }^{54,55}$ Polysaccharide A (PSA) from B. fragilis outer membrane vesicles was recently reported to tolerize dendritic cells through LC3-associated phagocytosis, which in turn increases IL-10 expression in gut Treg cells. ${ }^{57}$ Thus, a number of signals originating directly or indirectly from intestinal bacteria or the diet have been described to facilitate Treg cell differentiation or expansion in the gut.

While many of these mechanisms have been carefully studied on pTreg cell differentiation in vitro, their in vivo roles are not as well established. Recently, we have used bacteria-reactive colonic TCR Tg lines to begin to address these issues. ${ }^{33}$ For example, colonic TCR Tg cells expressing a dominant negative TGFßRII (dnTGFßRII) transgene to inhibit TGF $\beta$ signaling showed only a $50 \%$ blockage of $\mathrm{pTreg}$ cell generation, whereas a previous study examining non-mucosal associated pTreg cell generation showed a $90 \%$ decrease. ${ }^{43}$ As the dnTGF $\beta R I I$ transgene is a hypomorph, ${ }^{58}$ a requirement for TGF $\beta$ cannot be excluded. However, these data suggest that pTreg cell generation in the colon is not very sensitive to the level of TGF $\beta$ signaling, contrary to predictions based on prior in vitro and in vivo data. ${ }^{43}$ Although it has been suggested that the level of TGF $\beta$ is increased in the intestine, ${ }^{59}$ there is little evidence to suggest that the normal level of TGF $\beta$ in vivo is high enough to overcome the effect of dnTGF $\beta$ RII. ${ }^{33,60}$ Thus, one interpretation of these data is that TGF $\beta$ is not a singular or "master" factor that specifies pTreg cell selection, and that other signals in the colon are also important. 
Another key mechanism involved in pTreg cell selection is reported to involve CNS1, a region in the Foxp 3 locus ${ }^{34,35}$ that includes transcription factor binding sites downstream of TGF $\beta$ and retinoic acid signaling. ${ }^{61,62}$ Analysis of naive T cell differentiation using CNS1-deficient colonic TCR Tg cells revealed a strong inhibition of Foxp3-induction at seven days, but a gradual upregulation of Foxp 3 by two to five weeks, which could also be observed in polyclonal cells using Helios or Nrp-1. ${ }^{33}$ Thus, CNS1 appears to be important, but not essential, for pTreg cell generation.

Many more studies are required before the factors that affect the colonic Treg cell population are fully understood. One important question is whether these factors affect pTreg cell differentiation, versus expansion or gut homing of pre-existing Treg cells. In one paper, tTreg cell expansion, and not pTreg cell differentiation, was proposed to be the mechanism by which SCFAs via Gpr43 affect intestinal Treg cells. ${ }^{53}$ However, this issue is not easily addressed via analysis of polyclonal Treg cell numbers, requiring an approach where the kinetics of Treg cell induction and expansion can be monitored. ${ }^{33}$ Another major issue with experimental manipulations in the study of intestinal Treg cell generation are potential effects on the microbiota, both in terms of population composition but also bacterial gene expression, which is currently not often assessed. For example, NOD2-deficiency can lead to an expansion of B. vulgatus, ${ }^{63}$ which could directly or indirectly affect pTreg cell generation to $B$. vulgatus or other intestinal species by altering synthesis of SCFA, TLR signals for innate and adaptive immune cells, antigen presentation, and so forth. We think that this issue can be minimized by restricting the experimental manipulation to transferred T-cells, which constitute only a small fraction of $\mathrm{T}$-cells in the host. While the TCR transgenic transfer model is limited to the study of $\mathrm{T}$ cell intrinsic factors, it permits analysis of Foxp3-induction, proliferation, migration, and survival of $\mathrm{T}$-cells in a normal host during homeostasis.

\section{BACTERIAL SPECIES SPECIFICITY OF COLONIC T-CELLS}

The above discussion suggests that tolerance to intestinal bacteria is dependent on induction of Treg cells by intestinal bacteria. In addition to mechanisms such as SCFAs and TLR ligands that act through TCR-independent mechanisms, there is growing evidence that the development of colonic Treg cells is in response to species-specific bacterial antigens (Figure 1). This notion is consistent with TCR repertoire analyses of colonic T-cells during homeostasis. Our group found that colonic Treg cells utilize a different TCR repertoire than Treg cells from other secondary lymphoid organs, ${ }^{36}$ suggesting recognition of local colonic antigens. Another study showed that the colonic Treg cell repertoire was markedly affected by broad-spectrum antibiotics, ${ }^{26}$ consistent with bacterial antigens selecting the gut Treg cell population. Thus, these reports show that intestinal bacteria play an important role in shaping the TCR repertoire of colonic Treg cells during homeostasis. Another consideration is that the cLP may also contain pTreg cells with non-microbiota specificities and tTreg cells that are either specific to intestinal self-antigens or cross-react with bacterial antigens. Treg cells are observed in the cLP, albeit in reduced number, in germ free mice, ${ }^{22}$ indicating that nonmicrobiota-dependent Treg cells can home to and survive in colonic tissue.

Studies of individual bacterial species transferred into germfree mice support the concept that colonic Treg cells can be specific to bacteria. Both murine and human Clostridium species $^{22,47}$ (primarily from clusters IV, XIVa, and XVIII), but not all gut bacterial species, transferred to germ-free mice can induce increased frequencies of Treg cells. While the pro-Treg cell effects of Clostridium species may be both antigen-specific and non-specific, in vitro suppression by Treg cells from colonized mice were enhanced by inclusion of Clostridium, but not germ-free isolates with $\mathrm{CD}_{11 \mathrm{c}^{+}}$antigen presenting cells. ${ }^{47}$ While this is consistent with Clostridium TCR-specific Treg cell function, it is possible that Clostridium isolates enhanced Treg cell function via TCR-independent effects in this assay. In addition to Clostridium, a recent report identified several Bacteroides species that selectively induced increased percentages of $\mathrm{Nrp}^{10}$ Treg cells in germ-free mice upon monocolonization, ${ }^{64}$ although this may not be true of all Bacteroides species. ${ }^{22}$ While these mono-colonization data in germ-free mice do not provide direct proof that Treg cells recognize these bacteria in the context of a complex microbiota, they are consistent with the notion that certain bacterial species preferentially induce bacteria-specific pTreg cells.

Our studies of colon TCR specificities also support the existence of bacteria-specific colonic Treg cells. We screened 10 colonic Treg TCRs against a panel of murine bacteria in vitro. One Treg TCR, CT6, showed reactivity to a mouse unclassified Clostridium spp, ${ }^{36}$ which was unfortunately lost during cryopreservation. Subsequently, we have found that CT6 can recognize a human C. symbiosum isolate (unpublished). Another Treg TCR, CT7, was found to react to Parabacteroides distasonis and B. uniformis. ${ }^{36}$ However, recognition of Clostridium or Bacteroides spp. by these TCRs have not yet been confirmed in vivo.

On the whole, the bacterial species recognized by colonic Tconv and Treg cells in vivo in the setting of the complex microbiota in normal SPF mice remain unclear, in contrast with studies showing direct Th17 cell recognition of segmented filamentous bacteria (SFB) in the small intestine. ${ }^{65}$ The best characterized colonic TCR Tg model, CBir1, which recognizes a defined bacteria flagellin epitope, CBirl, is not reported to be activated during homeostasis. ${ }^{66}$ This is not an issue with the TCR Tg line, as analysis of polyclonal T-cells using a tetramer with the CBirl epitope shows that the majority of T-cells are in the phenotypically naive $\mathrm{CD} 44^{\mathrm{lo}}$ population. ${ }^{66}$ Interestingly, CBir1 flagellin falls within the Clostridium subphylum XIVa, which is associated with induction of Treg cells. ${ }^{22,47}$ These Clostridium species are common constituents of mouse microbiota, and the epitope is clearly present in most microbiota $^{67}$ (and unpublished observations). Thus, the availability of the epitope in the lumen does not always equate with antigen presentation to T-cells. 
The relative lack of conclusive data on T-cell specificity to gut bacteria is due to several issues. First, in vivo analysis of total Treg cell numbers or function is not proof of TCR recognition, as non-TCR-specific bacterial signals may be important. For example, SCFA have been shown to expand Treg cells in germfree mice. ${ }^{53-55}$ Similarly, purified PSA from $B$. fragilis has been shown to induce IL-10 on polyclonal Treg cells. ${ }^{68}$ Retinoic acid $^{48-50}$ and Ahr ligands ${ }^{69}$ might be expected to act similarly in an antigen-independent fashion. Second, the study of individual bacteria introduced into germ-free mice may result in their abnormal exposure to the immune system due to the availability of niches, which are normally filled by other bacterial species. $\mathrm{T}$ cell responses in mono-colonized mice would then need to be verified in more complex microbiota, requiring antigen-specific tools like transgenic mice or $\mathrm{MHC}$ tetramers. Third, culturing gut bacteria can be quite difficult. Most species require anaerobic techniques, and many remain unculturable. Fourth, the current protocols for in vitro culture may result in changes in bacterial antigen expression. Use of isolates with the same or similar taxonomy by $16 \mathrm{~S} \mathrm{rDNA}$ sequences may still miss isolate-specific changes in $\mathrm{T}$ cell epitopes. Finally, even if TCR recognition of in vitro grown bacteria is achieved, it remains possible that a different species actually provides the antigen to T-cells in vivo. In our limited experience, we have observed overlap of TCR reactivity to bacteria of similar taxonomy. For example, CT7 TCR recognizes epitopes from $B$. uniformis and $P$. distasonis, whereas DP1 TCR reacts with $B$. thetaiotaomicron, B. acidifaciens, B. vulgatus, B. sp. TP5, and P. goldsteinii. $^{36}$ Thus, there are a number of hurdles in identifying the species responsible for induction of bacteria-specific Treg cells in vivo.

\section{DYSREGULATION OF PTREG CELL DIFFERENTIATION IN THE COLON}

The current data suggest that bacteria-reactive Treg cells are often generated from naive T-cells. Gut perturbations may therefore affect $\mathrm{T}$ cell differentiation. Models of intestinal inflammation such as IL-10-deficiency are associated with increases in Th1 and Th17 cell frequencies and a decrease in Treg cell frequencies. ${ }^{70,71}$ On a monoclonal level, CBir1 TCR Tg cells, specific to commensal-derived flagellin, largely remain naive in healthy hosts but have been shown to develop into stable Th1 effector cells after Toxoplasma infection or into Th17 cells after DSS-mediated mucosal injury and colitis. ${ }^{66}$ Adoption of effector, rather than regulatory, T cell fate may be particularly relevant to the induction of immune responses against commensal antigens, which has been implicated in the pathogenesis of IBD. ${ }^{8,72,73}$ Consistent with this hypothesis, CT2/CT6 TCR sequences typically found in the Treg cell subset in normal mouse colons can be found in colonic effector Foxp $3^{-}$ $\mathrm{T}$ cell subsets in genetic backgrounds such as $I L-10^{-1-}$ and dnTGF $\beta$ RII mice that lead to spontaneous colitis. ${ }^{36}$ Treatment of mice with IL-10 R antibodies and DSS to induce a transient colitis resulted in differentiation of a portion of the transferred naive CT2/CT6 cells into ROR $\gamma \mathrm{t}^{+}$Th17 T-cells (unpublished, Chai \& Hsieh). In addition, transfer of naive CT2/CT6 T-cells into a $\mathrm{Rag1}^{-/-}$lymphopenic mice resulted in $\mathrm{Tbet}^{+}$Th1 differentiation. $^{33}$

The specific signals that disrupt the normal process of pTreg cell development to gut bacteria have not been extensively studied. In a polyclonal setting, expansion of already existing effector cells is likely during inflammation. The inflammatory milieu with altered cytokine balance can also promote differentiation of naive T-cells into effector phenotypes. Finally, intestinal changes that lead to bacterial antigens being acquired and presented by different antigen presenting cell (APC) subsets could affect pTreg vs effector T cell differentiation. ${ }^{74}$ Further work in this area could have therapeutic implications for intestinal inflammatory diseases.

\section{TREG CELLS CO-EXPRESSING CANONICAL EFFECTOR T CELL TRANSCRIPTIONS FACTORS}

While certain environments described above skew $\mathrm{T}$ cell development away from Treg towards effector subsets, normal Foxp $3^{+}$Treg cells can upregulate effector transcription factors such as Tbet, GATA3, and ROR $\gamma$ t to provide additional functional capacity. ${ }^{45}$ However, Treg expression of GATA3, the canonical transcription factor for Th2 cells, and Tbet for Th1 cells, appear to be independent of intestinal bacteria at homeostasis. GATA3 is expressed in about $15-20 \%$ of Treg cells in the colon and small intestine. ${ }^{75}$ GATA3 ${ }^{+}$Treg cells are equally abundant in germ-free versus SPF mice ${ }^{76}$ and express Helios, suggestive of tTreg lineage. ${ }^{76,77}$ Tbet $^{+}$Treg cells are even less frequent at around $5 \%$ in the mesenteric and peripheral lymph nodes and small intestine lamina propria. ${ }^{78}$ Thus, Treg cells co-expressing GATA3 or Tbet do not appear to play an integral part in the response to commensal bacteria during homeostasis.

By contrast, the colon is normally highly enriched in Treg cells that express the canonical Th17 transcription factor ROR $\gamma \mathrm{t}$ (30-80\%), which is expressed in only $10-20 \%$ of Treg cells in the small intestine. ${ }^{76,77,79,80}$ These cells are likely to be bacteria-induced pTreg cells, as they are: Helios ${ }^{\mathrm{lo}}$ and Nrp- $1^{\text {lo }}$, dramatically decreased in germ free and antibiotic-treated mice, and can be induced in germ-free mice to SPF percentages by several commensal species belonging to different phyla. ${ }^{76,77}$ ROR $\gamma \mathrm{t}^{+}$Treg cells express high levels of IL-10 and CTLA-4, ${ }^{76}$ consistent with activated Treg cells with increased immunoregulatory capability. ${ }^{80}$

The process by which ROR $\gamma \mathrm{t}^{+}$Treg cells are generated appears to be dependent on certain bacteria. A large study of single bacterial isolates from the human gastrointestinal tract revealed that only certain species induced ROR $\gamma$ t expression in Treg cells. ${ }^{77}$ Consistent with this observation, TCR repertoire analyses by our group using a fixed TCR $\beta$ model suggest that Foxp ${ }^{+} \mathrm{ROR}_{\mathrm{t}}{ }^{+}$Treg cells in the colon use TCRs largely distinct from ROR $\gamma \mathrm{t}^{-}$Treg cells and Th17 cells, although some overlap with Th17 cells was observed. ${ }^{79}$ However, another report suggested that TCR usage could be shared between ROR $\gamma \mathrm{t}^{+}$Treg and other subsets. ${ }^{80}$ The different results obtained between these two reports may be due to fixed TCR $\beta$-chain versus paired-chain TCR repertoire analysis in a 
fully polyclonal population, as well as sequencing of colon versus splenic and lymphoid organ T-cells. Nonetheless, this latter analysis is consistent with the observation that nonbacterial ovalbumin in an oral tolerance model induced OT-II cells to become ROR $\gamma \mathrm{t}^{+}$Treg cells in the small intestine, ${ }^{76}$ suggesting that the developmental signals do not need to be directly linked to the T-cell epitope. While additional studies are required to resolve these issues, the selectivity of certain gut bacteria to induce the ROR $\gamma \mathrm{t}^{+}$Treg cell population ${ }^{77}$ favors, but does not prove, the hypothesis that generation of these cells occurs in an antigen-specific context.

The microbiota-derived signals that drive generation of $\mathrm{ROR}_{\mathrm{t}}{ }^{+}$Treg cells are not well established. It has been suggested that T-cells first upregulate Foxp3 and then subsequently ROR $\gamma \mathrm{t}$ in both polyclonal and monoclonal T cell populations. ${ }^{77,79}$ The development of ROR $\gamma t$ after pTreg cell generation suggests that this may occur upon interaction with microbial-induced factors in the colon. Such factors may include IL- 6 and IL-23, although the studies were not entirely consistent. ${ }^{76,77}$ The microbial-derived signals that generate $\mathrm{ROR}_{\mathrm{t}}{ }^{+}$Treg cells in vivo therefore remain to be established. In addition, the temporal sequence of Foxp3 and then ROR $\gamma \mathrm{t}$ induction may only apply to a subset of cells. For example, it may be speculated that cells expressing TCRs that overlap with Th17 cells ${ }^{79}$ could arise from upregulating ROR $\gamma$ t first and then Foxp3.

Although ROR $\gamma \mathrm{t}$ is the canonical Th17 transcription factor, it is not clear that ROR $\gamma \mathrm{t}^{+}$Treg cells primarily exist to inhibit Th17-mediated inflammation. For example, the frequency of ROR $\gamma t$-expressing Treg cells ${ }^{76,77,79,80}$ in a tissue is inverse that of Th17 cells, which are found predominantly in the small intestine and not the colon. ${ }^{81}$ In addition, Treg cell-specific knockout of ROR $\gamma t$ has been reported to result in Th2mediated or Th1/Th17-mediated colitis, ${ }^{76,77}$ and not purely Th17-mediated disease. Future studies are therefore required to determine the specific mechanisms by which ROR $\gamma t$ expression facilitates Treg cell suppression of specific subsets of T effector cells in the colon.

\section{FUNCTION OF COLONIC TREG CELLS}

Intestinal Treg cells likely employ a number of mechanisms for regulating effector T-cell responses to gut bacteria, including cell surface proteins and cytokines (reviewed in ref. 17). One likely mechanism is the inhibition of APC function by CTLA-4, a transmembrane protein constitutively expressed in Treg cells. ${ }^{82}$ CTLA-4 binding to CD80/86 can prevent CD28 on effector T-cells from engaging and costimulating TCR activation. ${ }^{83}$ Treg cell CTLA-4 may also directly remove CD80/CD86 from APC membranes. ${ }^{84}$ Treg cell-mediated suppression via CTLA-4 appears to be important clinically, as an important side effect of CTLA-4 antibody therapy for human malignancy is the development of colitis. ${ }^{85}$

Treg cells also secrete pro-tolerogenic cytokines such as TGF $\beta{ }^{86}$ Deletion of TGF $\beta 1$ from T-cells results in spontaneous autoimmunity, albeit delayed in comparison with a germ-line knockout. ${ }^{87}$ While one might have predicted that Treg cell- derived TGF $\beta 1$ was primarily affected by $\mathrm{CD} 44^{\mathrm{Cre}}$-mediated

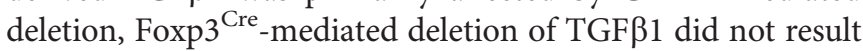
in development of spontaneous autoimmunity or colitis. ${ }^{88}$ However, an effect of TGF $\beta 1$ was observed in the T cell transfer model of colitis into lymphopenic hosts, ${ }^{87}$ suggesting that certain experimental conditions could reveal a role for Treg cell-derived TGF $\beta$. Thus, these data demonstrate that while TGF $\beta$ production by Treg cells may play a role, it is not essential for preserving normal gut homeostasis.

IL-10 is another important immuno-regulatory cytokine in the gut. Genetic polymorphisms of IL-10 and IL-10R in humans are associated with development of IBD ${ }^{89,90}$ and IL-10 deficient mice develop spontaneous colitis. ${ }^{91}$ T-cells are an important source of IL-10, ${ }^{92}$ with important contributions from both Treg and non-Treg cells. ${ }^{93}$ Notably, of the CD4 ${ }^{+}$ T-cell population, colonic Treg cells exhibit the greatest production of IL-10 based on the use of reporters, ${ }^{94}$ and the expression of IL-10 is associated with co-expression of ROR $\gamma \mathrm{t} .{ }^{76}$ Induction of IL-10 in gut Treg cells has been reported to occur with microbiota-derived factors such as B. fragilisderived PSA via a T-cell intrinsic TLR2-dependent mechanism. ${ }^{68}$ In summary, there is considerable evidence that gut bacteria induce IL-10 expressing pTreg cells in the colon, and that Treg cell production of IL-10 is necessary to preserve gut homeostasis.

However, none of the Treg cell-derived factors such as CTLA- 4 or regulatory cytokines like IL-10 appear to be directed at effector T-cells in an antigen-specific manner. Rather, Treg cell inhibition of antigen-specific effector cells could occur via effects on APCs. A recent study using multiplex quantitative imaging showed that Treg cells cluster with $\mathrm{CD} 11 \mathrm{c}^{+} \mathrm{MHCII}^{\mathrm{hi}}$ APCs in lymphoid tissue and that CTLA-4 expression is increased in clustered versus non-clustered Treg cells. ${ }^{95}$ Expression of the TCR was required on Treg cells for Treg cell/APC clustering. Thus, these data provide evidence that TCR activation of gut bacteria-specific pTreg cells generate a local microenvironment that is anti-inflammatory via CTLA-4 and IL-10 interactions with dendritic cells, thereby inhibiting effector cells specific to bacterial antigens that may also be presented on that APC.

\section{ANTIGEN-SPECIFICITY OF IgA}

The specificity of gut IgA responses may provide important clues for understanding $\mathrm{T}$ cell responses in the colon. IgA is known to be induced against toxins and pathogenic bacteria, ${ }^{96}$ but it has become clear that IgA is also induced by normal intestinal bacteria during homeostasis. ${ }^{97}$ Unexpectedly, this recognition may actually facilitate the uptake and presentation of bacteria or other antigens within the intestinal lumen to the adaptive immune system. ${ }^{97}$ Thus, IgA responses may reflect adaptive immune responses to intestinal bacterial antigens.

One important issue, though, is that IgA specificity cannot be directly equated with T-cell antigen-recognition, as both $\mathrm{T}$-dependent (TD) and T-independent (TI) IgA responses occur. ${ }^{98,99}$ Some studies have suggested that the majority of IgA in the gut is TD, as the level of free IgA in T-cell-deficient mice is 
1/5th that of mice with T-cells. ${ }^{100}$ In addition, sequencing studies showed that a large portion of gut IgA has somatic hypermutation, ${ }^{97,101}$ supporting a substantial role for $\mathrm{T}$ follicular helper (Tfh) cells in gut IgA induction. By contrast, studies assessing IgA reactivity to bacteria using flow cytometry of T-cell-deficient mice have varied in their interpretation as to whether TI IgA represents a major ${ }^{102}$ versus minor ${ }^{103}$ part of the $\mathrm{B}$ cell response to commensal bacteria. However, the flow cytometry assay does not assess reactivity to secreted bacterial antigens or outer-membrane vesicles, which could be important targets of TD IgA. Thus, the relative contribution of TD versus TI IgA in response to commensal bacteria during homeostasis is incompletely understood.

TD IgA to surface bacterial antigens seen by bacterial flow cytometry may occur primarily to immunostimulatory bacteria strong enough to induce Tfh responses. ${ }^{97}$ For example, IgA responses to bacteria obtained from co-housing with inflammasome-deficient $\left(\mathrm{Asc}^{-/-}\right)$mice occurred only in T-cellsufficient mice ${ }^{103}$ and were specific to Prevotellaceae, Helicobacter sp. flexspira, and SFB-all potential drivers of intestinal inflammation. ${ }^{104-106}$ In other studies, two groups observed that $\mathrm{SFB}^{107}$ and Mucispirillum, ${ }^{108}$ both bacteria that tightly adhere to the epithelium, induce TD IgA. ${ }^{102,103}$ In studies of fecal bacteria from IBD patients, species that were preferentially $\mathrm{IgA}^{+}$, and not IgA ${ }^{-}$, facilitated DSS-mediated colitis in mice. ${ }^{103}$ Similarly, a study of human undernourished children with enteropathy revealed IgA-responses to Enterobacteriaceae, whose pathogenicity was confirmed in mice. ${ }^{109}$ Finally, pathogenic secreted bacterial factors like cholera toxin specifically require Th17 cells for IgA induction. ${ }^{110}$ Thus, bacteria with increased immunostimulatory potential such as pathobionts may be speculated to induce TD IgA responses.

In addition to these analyses, TCR Tg studies have supported a role for anti-bacterial TD IgA. CBir1 Tg Treg cells induce flagellin-specific IgA molecules, when transferred into T-celldeficient mice. ${ }^{111}$ Similarly, we have observed that DP1 transgenic T-cells, which recognize Bacteroidaceae species in vitro, ${ }^{36}$ can also induce IgA responses against those species when adoptively transferred into T cell-deficient mice (unpublished). One caveat of these studies is that they are done in lymphopenic mice, which may show altered $T$ cell development as well as intestinal permeability to bacteria (Figure 2). Nonetheless, these data directly demonstrate that antigenspecific $\mathrm{T}$ cell responses can induce bacteria-specific IgA. In summary, there has been significant progress in understanding the specificity of intestinal IgA. However, much remains to be learned about the characteristics of the bacteria that result in $\mathrm{T}$ and $\mathrm{B}$ cell responses during homeostasis and the Thelper subset responses they elicit.

\section{ROLE FOR TREG CELLS IN INDUCTION OR SUPPRESSION OF IgA}

With the exception of SFB, there is a relative dearth of studies that integrate both $\mathrm{T}$ and $\mathrm{B}$ cell reactivity to intestinal bacteria at homeostasis. For example, Clostridium spp. induce Treg cell responses, ${ }^{22}$ but the IgA responses are not characterized in normal hosts. Several Lactobacillus species increase the percentage of Treg cells in the colon and spleen, ${ }^{104,112}$ but Lactobacillus-specific IgA appears to be TI. ${ }^{102,103}$ In contrast, Mucispirillum species induce TD $\operatorname{IgA},{ }^{102,103}$ but the $\mathrm{T}$ cell response to Mucispirillum during homeostasis has not been clearly established. One example in which data are available for both $\mathrm{T}$ and $\mathrm{B}$ cell responses is Bacteroides thetaiotamicron, which can induce $\operatorname{IgA} \mathrm{A}^{113}$ and expansion of colonic ROR $\gamma \mathrm{t}^{+}$ pTreg cells ${ }^{77}$ when monocolonized in germ-free hosts. By combining the results of these two different studies, it could be hypothesized that Treg cell responses drive IgA induction during homeostasis.

A role for Treg cells in IgA induction has been proposed. In a $\mathrm{T}$ cell transfer model into lymphopenic mice, co-transfer of Foxp $3^{+}$Treg cells is necessary to limit colitis, maintain intestinal microbial diversity, and induce IgA. ${ }^{114}$ This process appeared to result from loss of Foxp 3 in a portion of the transferred Treg cells, which then upregulate Tfh markers such as Bcl6, IL-21, CD40L, and CXCR5. ${ }^{115}$ Another group used the CBir1 TCR Tg line to show that transfer of Treg cells into TCR-deficient mice preferentially induced CBir1-specific IgA. ${ }^{111}$ In addition, depletion of Treg cells using anti-CD25 led to a marked drop in anti-CBir1 IgA, suggesting that Treg cells may directly induce IgA via TGF $\beta$. However, the use of lymphopenic models may result in loss of Treg cell stability, ${ }^{116}$ leading to the transdifferentiation of Treg into Foxp $3^{-}$Tfh cells, which may not occur in normal hosts. In addition, the use of anti-CD25 antibodies may kill activated T-cells or lead to gut inflammation, which may alter the range of antigens presented to the immune system. ${ }^{66}$ Thus, while there is intriguing data suggesting that gut Treg cells induce IgA, either directly or serving as precursors for Foxp3 ${ }^{-}$Tfh cells, this Treg cell function during normal homeostasis requires further investigation.

Moreover, it has been argued that Treg cells actually inhibit B cell germinal center responses via the $\mathrm{T}$ follicular regulatory (Tfr) cell subset. In the spleen, Tfr cells have been shown to develop from Foxp ${ }^{+}$tTreg cells, maintain expression of Foxp3, and upregulate expression of Tfh markers such as PD-1, CXCR5, and Bcl6. ${ }^{117}$ However, Tfr cells do not express CD40L or the Tfh cytokines IL-21 and IL-4, and function to limit the number of Tfh cells in germinal centers as well as inhibit the non-antigen-specific selection of B cells to prevent autoantibody formation. ${ }^{117-119}$ Consistent with these observations in non-mucosal sites, Peyer's patch (PP) Foxp ${ }^{+}$germinal center Tfr cells were necessary to produce more specific, affinitymatured IgA. ${ }^{114}$ Defective Tfr function resulted in a larger percentage of fecal bacteria that stained $\operatorname{IgA}{ }^{+}$, suggesting that Tfr responses are more important in the gut for limiting IgA induction, rather than directly inducing IgA responses themselves.

Interestingly, $\mathrm{CNS}^{-/-}$Foxp3 mice, which show decreased induction of pTreg cells, develop plasmacytic enteritis characterized by increased serum antibodies against small intestine, large intestine, and chow antigens, ${ }^{34}$ which could hint at the necessity of antigen-specific Tfr cells to maintain intestinal homeostasis. However, it can be difficult to dissect in this model 

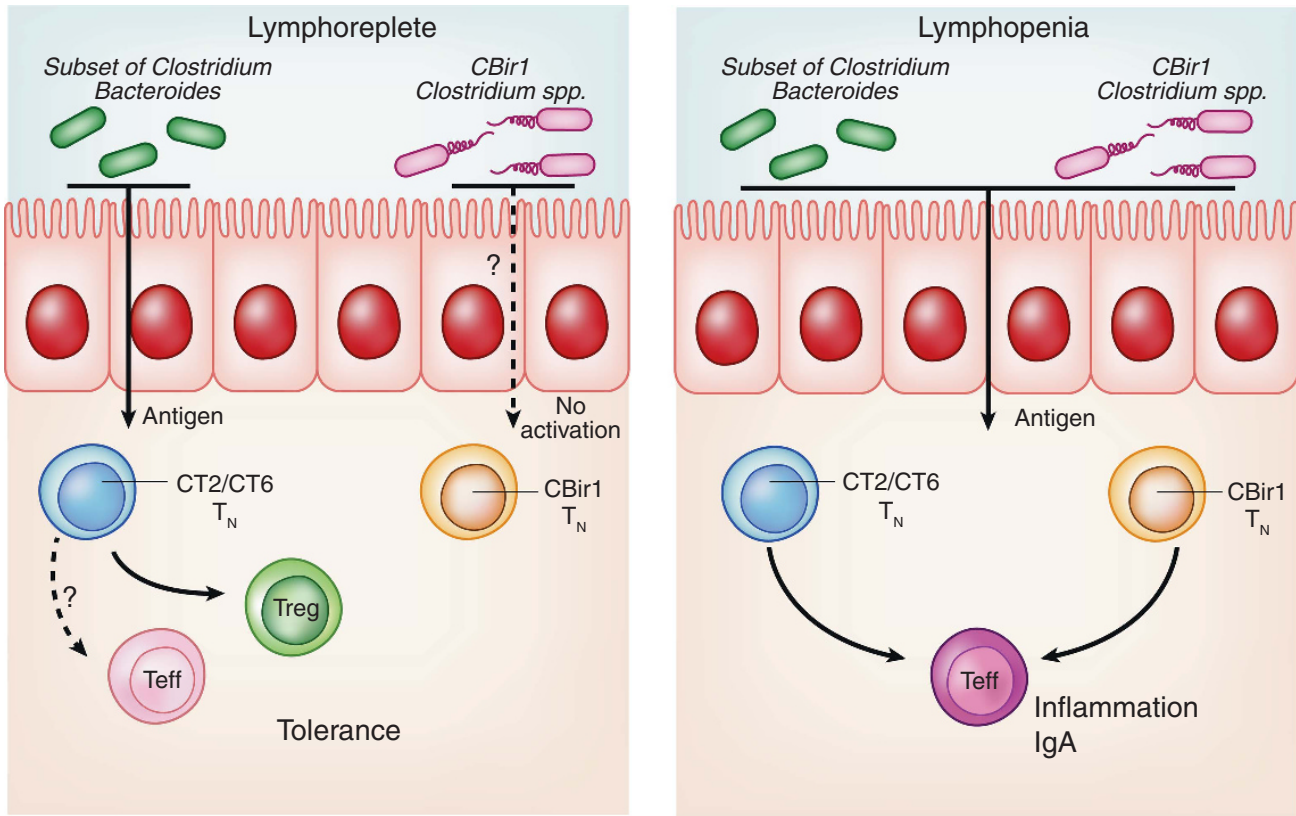

Figure 2 Alternative regulation of intestinal bacterial antigen presentation and T cell fate in lymphoreplete vs. lymphopenic mice. In lymphoreplete mice, commensal spp. induce naive colonic TCR transgenic CT2/CT6 cells into Treg cells. On the basis of in vitro and in vivo studies, it is predicted that select Bacteroides and Clostridium spp. induce antigen-specific Treg cells in lymphoreplete mice. However, naive CT2/CT6 T-cells develop into effector T-cells in $R a g 1^{-/-}$lymphopenic mice. In contrast to the behavior of of CT2/CT6, CBir1 antigen, a component of flagella present in many gut bacteria species including those in Clostridium subphylum XIVa, does not activate T-cells or induce differentiation of Treg cells in lymphoreplete mice. However, these antigens are presented in lymphopenic mice leading to $T$ cell activation and IgA production. Mucosal injury or infection will also lead to antigen presentation to CBir1 reactive T-cells. In these environments, CBir1 reactive T-cells become effector, and not Treg, cells.

whether this is due to Treg cells controlling effector T-cell activation versus $\mathrm{Tfh}$ cells in the germinal center reaction. Thus, future studies are required to determine whether gut Treg cells promote and/or inhibit IgA responses.

In brief, the role of Treg cells in TD IgA remains unclear. A straightforward hypothesis is that bacteria induce Treg cells, which, via expression of TGF $\beta$, facilitate IgA class switch. However, it may be possible that Treg cells do not induce TD IgA. In this case, it may be possible that bacteria could induce a small fraction of effector T-cells that stimulate IgA production, which may be subsequently regulated by Treg cell-derived Tfr cells. Thus, new studies are required to understand the concomitant $\mathrm{T}$ and $\mathrm{B}$ cell response to intestinal bacteria at homeostasis.

\section{CONCLUSIONS}

In summary, there has been considerable progress recently in our understanding of adaptive immune responses to colonic bacteria during homeostasis. First, it is clear that intestinal bacteria play a crucial role in educating the colonic immune system, with distinct effects on the TCR repertoire. TD IgA responses are also induced, although it has not been established whether this occurs in the colon or elsewhere. Second, the $\mathrm{T}$ cell response to colonic bacteria can result in naive T-cell differentiation into pTreg cells. Commensal bacteria appear to facilitate this process via provision of antigens and generation of metabolites such as SCFA or inducing TGF $\beta$ production by epithelial cells. Third, pTreg cell responses can be the dominant outcome of naive T-cell differentiation in vivo, making it a bone fide peripheral $\mathrm{T}$ helper subset. Finally, bacteria-induced pTreg cells are necessary in vivo to prevent colitis.

However, these data also raise important issues. For example, what are the bacterial specificities of colonic Treg cells during homeostasis in the context of a normal microbiota (Figure 1)? If IgA specificity is a guide, we might predict that there will be relatively few bacterial species that induce pTreg cell responses in a normal host. This would be consistent with the observation that CBir1 TCR Tg cells do not see their antigen under homeostatic conditions, ${ }^{66}$ even though it is clear that the antigen is relatively abundant in the lumen. We speculate that this means that the mucous/mucosa layer functions as a "wall" to prevent many/most bacterial antigens access to immune cells (Figure 2). This contrasts with the small intestine, where oral administration of ovalbumin results in antigen presentation to T-cells and Treg cell induction. ${ }^{120,121}$ This is further supported by the recent observation that the majority of small intestinal pTreg cells are induced by dietary antigens. ${ }^{122}$ Yet, it is evident that commensal bacteria have a tremendous impact on the colon TCR repertoire, suggesting that those bacteria that do get through are immunodominant over self-antigen presentation. One possible explanation is that outer membrane vesicles ${ }^{123}$ or soluble antigens ${ }^{124}$ may have easier access to APCs than bacterial-associated antigens such as flagellin. Nonetheless, we predict that only a small fraction of intestinal bacterial antigens is presented to the adaptive immune system in the colon. 
Another issue is the use of lymphopenic mice for the study of intestinal tolerance (Figure 2). While it has been the classic model by which to study the role of Treg cells in the colon, ${ }^{125}$ our study suggests that pTreg cell differentiation is markedly perturbed in this environment. ${ }^{33}$ This may be due to abnormal barrier function, which results in bacterial translocation into the lamina propria, mesenteric lymph nodes, and spleen. ${ }^{11}$ This may facilitate increased proliferation ${ }^{126}$ and induce an unbalanced cytokine environment that favors effector $\mathrm{T}$ cell differentiation of bacteria-specific T-cells. Nonetheless, it may be possible in the future to establish conditions that "normalize" the lymphopenic model by adding in sub-therapeutic amounts of polyclonal Treg cells ${ }^{39}$ or other manipulations such as anti-cytokine antibody, thereby permitting the study of commensal-specific pTreg cell differentiation and function in a setting where the antigen-specificities can be experimentally controlled.

While most of this review has focused on Treg cells, the role of effector T-cells during homeostasis is not well characterized. As noted above, the observed bacterial translocation from the intestinal lumen to secondary immune organs in lymphopenic mice suggests that $\mathrm{T}$-cells are required for full barrier function. However, other than Th17 cells reactive to SFB, identification of effector cells reactive to colonic bacteria during homeostasis has not been successful. ${ }^{36}$ One possibility is that Treg cells, perhaps via ROR $\gamma \mathrm{t}$, are capable of generating the signals required for epithelial homeostasis. Another is that commensal bacteria may induce, along with pTreg cells, some effector T-cells that acts to improve barrier function and limit bacterial invasion. The small fraction of bacteria-specific effector T-cells would be constrained by Treg cells. Like self-reactive effector T-cells, their presence may only be recognized when Treg cells are depleted. ${ }^{127}$ A combined effector:Treg cell response to commensal antigens would be analogous to concomitant immunity such as that observed with Treg cells in Leishmania infection. ${ }^{128}$ Future studies will be required to determine the role of effector T-cells for intestinal homeostasis to commensal bacteria.

Finally, the type and extent of antigen-specific $\mathrm{T}$ cell responses involved in IgA induction is poorly characterized during homeostasis. It is unknown whether induction of IgA to commensal bacteria even occurs in the colon, as it may occur in more proximal parts of the intestine which have a thinner mucous layer and more defined antigen uptake mechanisms. Another question is whether Treg, Th17, or another subset is involved in IgA induction to commensal bacteria. Thus, many questions remain regarding gut bacterial interactions with the adaptive immune system.

\section{ACKNOWLEDGMENTS}

C.S.H. is supported by NIH grants R01 DK094995 and R21 Al097535, the CCFA, and the Burroughs Wellcome Fund. E.V.R.-G. is supported by NIH grant F30 DK111071-01. E.V.R.-G. and S.R. are supported by NIH grant T32 GM007200-42.

\section{AUTHOR CONTRIBUTIONS}

E.V.R.-G., S.R., and C.-S.H. contributed to the planning, writing, and editing of this review.

\section{DISCLOSURE}

The authors declared no conflict of interest.

c) 2017 Society for Mucosal Immunology

\section{REFERENCES}

1. Gillbert, J.A. et al. Microbiome-wide association studies link dynamic microbial consortia to disease. Nature 535, 94-103 (2016).

2. Hand, T.W. The role of the microbiota in shaping infectious immunity. Trends Immunol. 37, 647-658 (2016).

3. Leslie, J.L. \& Young, V.B. The rest of the story: the microbiome and gastrointestinal infections. Curr. Opin. Microbiol. 23, 121-125 (2015).

4. Flint, H.J., Scott, K.P., Louis, P. \& Duncan, S.H. The role of the gut microbiota in nutrition and health. Nat. Rev. Gastroenterol. Hepatol. 9, 577-589 (2012).

5. Blanton, L.V., Barratt, M.J., Charbonneau, M.R., Ahmed, T. \& Gordon, J.I. Childhood undernutrition, the gut microbiota, and microbiota-directed therapeutics. Science 352, 1533 (2016).

6. Belkaid, Y. Regulatory T cells and infection: a dangerous necessity. Nat. Rev. Immunol. 7, 875-888 (2007).

7. Maizels, R.M. \& Smith, K.A. Regulatory T cells in infection. Adv. Immunol. 112, 73-136 (2011)

8. Maloy, K.J. \& Powrie, F. Intestinal homeostasis and its breakdown in inflammatory bowel disease. Nature 474, 298-306 (2011).

9. Alexander, K.L., Targan, S.R. \& Elson, C.O. 3rd Microbiota activation and regulation of innate and adaptive immunity. Immunol. Rev. 260, 206-220 (2014).

10. Honda, K. \& Littman, D.R. The microbiota in adaptive immune homeostasis and disease. Nature 535, 75-84 (2016).

11. Mombaerts, P., Mizoguchi, E., Grusby, M.J., Glimcher, L.H., Bhan, A.K. \& Tonegawa, S. Spontaneous development of inflammatory bowel disease in T cell receptor mutant mice. Cell 75, 274-282 (1993).

12. Veazey, R.S. et al. Gastrointestinal tract as a major site of CD4 + T cell depletion and viral replication in SIV infection. Science 280, 427-431 (1998).

13. Brenchley, J.M. \& Douek, D.C. HIV infection and the gastrointestinal immune system. Mucosal Immunol. 1, 23-30 (2008).

14. Vallance, B.A., Deng, W., Knodler, L.A. \& Finlay, B.B. Mice lacking Tand B lymphocytes develop transient colitis and crypt hyperplasia yet suffer impaired bacterial clearance during Citrobacter rodentium infection. Infect. Immun. 70, 2070-2081 (2002).

15. Simmons, C.P. et al. Central role for B lymphocytes and CD4 + T cells in immunity to infection by the attaching and effacing pathogen Citrobacter rodentium. Infect. Immun. 71, 5077-5086 (2003).

16. Maloy, K.J. \& Powrie, F. Regulatory T cells in the control of immune pathology. Nat. Immunol. 2, 816-822 (2001).

17. Josefowicz, S.Z., Lu, L.F. \& Rudensky, A.Y. Regulatory T cells: mechanisms of differentiation and function. Annu. Rev. Immunol. 30, 531-564 (2012).

18. Roncarolo, M.G., Gregori, S., Bacchetta, R. \& Battaglia, M. Tr1 cells and the counter-regulation of immunity: natural mechanisms and therapeutic applications. Curr. Top. Microbiol. Immunol. 380, 39-68 (2014).

19. Sun, M., He, C., Cong, Y. \& Liu, Z. Regulatory immune cells in regulation of intestinal inflammatory response to microbiota. Mucosal Immunol. 8, 969-978 (2015).

20. Sarrabayrouse, G., Alameddine, J., Altare, F. \& Jotereau, F. Microbiotaspecific CD4CD8alphaalpha tregs role in intestinal immune homeostasis and implications for IBD. Front. Immunol. 6, 522 (2015).

21. Sujino, T. et al. Tissue adaptation of regulatory and intraepithelial CD4(+) T cells controls gut inflammation. Science 352, 1581-1586 (2016).

22. Atarashi, $\mathrm{K}$. et al. Induction of colonic regulatory $\mathrm{T}$ cells by indigenous Clostridium species. Science 331, 337-341 (2011).

23. Geuking, M.B. et al. Intestinal bacterial colonization induces mutualistic regulatory T cell responses. Immunity 34, 794-806 (2011).

24. Klein, L., Kyewski, B., Allen, P.M. \& Hogquist, K.A. Positive and negative selection of the T cell repertoire: what thymocytes see (and don't see). Nat. Rev. Immunol. 14, 377-391 (2014). 
25. Lee, H.M., Bautista, J.L., Scott-Browne, J., Mohan, J.F. \& Hsieh, C.S. A broad range of self-reactivity drives thymic regulatory $T$ cell selection to limit responses to self. Immunity 37, 475-486 (2012).

26. Cebula, A. et al. Thymus-derived regulatory Tcells contribute to tolerance to commensal microbiota. Nature 497, 258-262 (2013).

27. Thornton, A.M. et al. Expression of Helios, an Ikaros transcription factor family member, differentiates thymic-derived from peripherally induced Foxp3 + T regulatory cells. J. Immunol. 184, 3433-3441 (2010).

28. Weiss, J.M. et al. Neuropilin 1 is expressed on thymus-derived natural regulatory $\mathrm{T}$ cells, but not mucosa-generated induced Foxp3 + T reg cells. J. Exp. Med. 209, S1721 (2012).

29. Yadav, M. et al. Neuropilin-1 distinguishes natural and inducible regulatory T cells among regulatory T cell subsets in vivo. J. Exp. Med. 209, S1711-S1719 (2012).

30. Akimova, T., Beier, U.H., Wang, L., Levine, M.H. \& Hancock, W.W. Helios expression is a marker of Tcell activation and proliferation. PLOS ONE 6, e24226 (2011).

31. Gottschalk, R.A., Corse, E. \& Allison, J.P. Expression of Helios in peripherally induced Foxp3 + regulatory T cells. J. Immunol. 188, 976-980 (2012).

32. Szurek, E. et al. Differences in expression level of helios and neuropilin-1 do not distinguish thymus-derived from extrathymically-induced CD4 + Foxp3 + regulatory T cells. PLOS ONE 10, e0141161 (2015).

33. Nutsch, K. et al. Rapid and efficient generation of regulatory $T$ cells to commensal antigens in the periphery. Cell Rep. 17, 206-220 (2016).

34. Josefowicz, S.Z. et al. Extrathymically generated regulatory Tcells control mucosal TH2 inflammation. Nature 482, 395-399 (2012).

35. Zheng, Y., Josefowicz, S., Chaudhry, A., Peng, X.P., Forbush, K. \& Rudensky, A.Y. Role of conserved non-coding DNA elements in the Foxp3 gene in regulatory T-cell fate. Nature 463, 808-812 (2010).

36. Lathrop, S.K. et al. Peripheral education of the immune system by colonic commensal microbiota. Nature 478, 250-254 (2011).

37. Stadinski, B.D. et al. Hydrophobic CDR3 residues promote the development of self-reactive T cells. Nat. Immunol. 17, 946-955 (2016).

38. Haribhai, D. et al. A central role for induced regulatory T cells in tolerance induction in experimental colitis. J. Immunol. 182, 3461-3468 (2009).

39. Haribhai, D. et al. A requisite role for induced regulatory Tcells in tolerance based on expanding antigen receptor diversity. Immunity 35, 109-122 (2011).

40. Legoux, F.P. et al. CD4 + Tcell tolerance to tissue-restricted self antigens is mediated by antigen-specific regulatory $T$ cells rather than deletion. Immunity 43, 896-908 (2015).

41. Burzyn, D. et al. A special population of regulatory $T$ cells potentiates muscle repair. Cell 155, 1282-1295 (2013).

42. Gottschalk, R.A., Corse, E. \& Allison, J.P. TCR ligand density and affinity determine peripheral induction of Foxp3 in vivo. J. Exp. Med. 207, 1701-1711 (2010).

43. Kretschmer, K., Apostolou, I., Hawiger, D., Khazaie, K., Nussenzweig, M.C. \& von Boehmer, $\mathrm{H}$. Inducing and expanding regulatory T cell populations by foreign antigen. Nat. Immunol. 6, 1219-1227 (2005).

44. Pantoja-Feliciano, I.G. et al. Biphasic assembly of the murine intestinal microbiota during early development. ISME J. 7, 1112-1115 (2013).

45. Tanoue, T., Atarashi, K. \& Honda, K. Development and maintenance of intestinal regulatory T cells. Nat. Rev. Immunol. 16, 295-309 (2016).

46. Chen, W. et al. Conversion of peripheral CD4 + CD25- naive T cells to CD4 + CD25 + regulatory T cells by TGF-beta induction of transcription factor Foxp3. J. Exp. Med. 198, 1875-1886 (2003).

47. Atarashi, K. et al. Treg induction by a rationally selected mixture of Clostridia strains from the human microbiota. Nature 500, 232-236 (2013).

48. Benson, M.J., Pino-Lagos, K., Rosemblatt, M. \& Noelle, R.J. All-trans retinoic acid mediates enhanced Treg cell growth, differentiation, and gut homing in the face of high levels of co-stimulation. J. Exp. Med. 204 1765-1774 (2007).

49. Mucida, D. et al. Retinoic acid can directly promote TGF-beta-mediated Foxp3(+) Treg cell conversion of naive T cells. Immunity 30, 472-473 (2009).

50. Nolting, J. et al. Retinoic acid can enhance conversion of naive into regulatory T cells independently of secreted cytokines. J. Exp. Med. 206, 2131-2139 (2009).
51. Matteoli, G. et al. Gut CD103 + dendritic cells express indoleamine 2,3dioxygenase which influences Tregulatory/Teffector cell balance and oral tolerance induction. Gut 59, 595-604 (2010).

52. Munn, D.H. \& Mellor, A.L. Indoleamine 2,3 dioxygenase and metabolic control of immune responses. Trends Immunol. 34, 137-143 (2013).

53. Smith, P.M. et al. The microbial metabolites, short-chain fatty acids, regulate colonic Treg cell homeostasis. Science 341, 569-573 (2013).

54. Furusawa, Y. et al. Commensal microbe-derived butyrate induces the differentiation of colonic regulatory T cells. Nature 504, 446-450 (2013).

55. Arpaia, N. et al. Metabolites produced by commensal bacteria promote peripheral regulatory T-cell generation. Nature 504, 451-5 (2013).

56. Singh, N. et al. Activation of Gpr109a, receptor for niacin and the commensal metabolite butyrate, suppresses colonic inflammation and carcinogenesis. Immunity 40, 128-139 (2014).

57. Chu, H. et al. Gene-microbiota interactions contribute to the pathogenesis of inflammatory bowel disease. Science 352, 1116-1120 (2016).

58. Ishigame, H., Mosaheb, M.M., Sanjabi, S. \& Flavell, R.A. Truncated form of TGF-betaRIl, but not its absence, induces memory CD8 $+\mathrm{T}$ cell expansion and lymphoproliferative disorder in mice. J. Immunol. 190, 6340-6350 (2013).

59. Konkel, J.E. \& Chen, W. Balancing acts: the role of TGF-beta in the mucosal immune system. Trends Mol. Med. 17, 668-676 (2011).

60. Feuerer, M., Hill, J.A., Kretschmer, K., von Boehmer, H., Mathis, D. \& Benoist, C. Genomic definition of multiple ex vivo regulatory T cell subphenotypes. Proc Natl Acad. Sci. USA 107, 5919-5924 (2010).

61. Xu, L., Kitani, A., Stuelten, C., McGrady, G., Fuss, I. \& Strober, W. Positive and negative transcriptional regulation of the Foxp3 gene is mediated by access and binding of the Smad3 protein to enhancer I. Immunity 33, 313-325 (2010).

62. Tone, Y., Furuuchi, K., Kojima, Y., Tykocinski, M.L., Greene, M.I. \& Tone, M. Smad3 and NFAT cooperate to induce Foxp3 expression through its enhancer. Nat. Immunol. 9, 194-202 (2008).

63. Ramanan, D., Tang, M.S., Bowcutt, R., Loke, P. \& Cadwell, K. Bacterial sensor Nod2 prevents inflammation of the small intestine by restricting the expansion of the commensal Bacteroides vulgatus. Immunity 41, 311-324 (2014).

64. Faith, J.J., Ahern, P.P., Ridaura, V.K., Cheng, J. \& Gordon, J.I. Identifying gut microbe-host phenotype relationships using combinatorial communities in gnotobiotic mice. Sci. Transl. Med. 6, 220ra-211 (2014).

65. Yang, Y. et al. Focused specificity of intestinal T17 cells towards commensal bacterial antigens. Nature 510, 152-156 (2014).

66. Hand, T.W. et al. Acute gastrointestinal infection induces long-lived microbiota-specific T cell responses. Science 337, 1553-1556 (2012).

67. Pabst, O. \& Hornef, M. Gut microbiota: a natural adjuvant for vaccination. Immunity 41, 349-351 (2014).

68. Round, J.L. \& Mazmanian, S.K. Inducible Foxp3 + regulatory T-cell development by a commensal bacterium of the intestinal microbiota. Proc. Natl Acad. Sci. USA 107, 12204-12209 (2010).

69. Quintana, F.J. et al. Control of $\mathrm{T}(\mathrm{reg})$ and $\mathrm{T}(\mathrm{H}) 17$ cell differentiation by the aryl hydrocarbon receptor. Nature 453, 65-71 (2008).

70. Keubler, L.M., Buettner, M., Hager, C. \& Bleich, A. A multihit model: colitis lessons from the interleukin-10-deficient mouse. Inflamm. Bowel Dis. 21, 1967-1975 (2015).

71. Chaudhry, A. et al. Interleukin-10 signaling in regulatory T cells is required for suppression of Th17 cell-mediated inflammation. Immunity 34, 566-578 (2011).

72. Elson, C.O. \& Cong, Y. Host-microbiota interactions in inflammatory bowel disease. Gut Microb. 3, 332-344 (2012).

73. Jostins, L. et al. Host-microbe interactions have shaped the genetic architecture of inflammatory bowel disease. Nature 491, 119-124 (2012).

74. Cummings, R.J. et al. Different tissue phagocytes sample apoptotic cells to direct distinct homeostasis programs. Nature 539, 565-569 (2016).

75. Wohlfert, E.A. et al. GATA3 controls Foxp3(+ ) regulatory Tcell fate during inflammation in mice. J. Clin. Investig. 121, 4503-4515 (2011).

76. Ohnmacht, C. et al. The microbiota regulates type 2 immunity through RORgammat(+) T cells. Science 349, 989-993 (2015). 
77. Sefik, E. et al. Individual intestinal symbionts induce a distinct population of RORgamma $(+)$ regulatory T cells. Science 349, 993-997 (2015).

78. Yu, F., Sharma, S., Edwards, J., Feigenbaum, L. \& Zhu, J. Dynamic expression of transcription factors T-bet and GATA-3 by regulatory T cells maintains immunotolerance. Nat. Immunol. 16, 197-206 (2015).

79. Solomon, B.D. \& Hsieh, C.S. Antigen-specific development of mucosal Foxp3 + RORgammat $+T$ cells from regulatory $T$ cell precursors. J. Immunol. 197, 3512-3519 (2016).

80. Yang, B.H. et al. Foxp3(+) T cells expressing RORgammat represent a stable regulatory T-cell effector lineage with enhanced suppressive capacity during intestinal inflammation. Mucosal Immunol. 9, 444-457 (2016).

81. Ivanov, II et al. The orphan nuclear receptor RORgammat directs the differentiation program of proinflammatory IL-17 + T helper cells. Cell 126, 1121-1133 (2006).

82. Wing, K. et al. CTLA-4 control over Foxp3 + regulatory T cell function. Science 322, 271-275 (2008).

83. Esensten, J.H., Helou, Y.A., Chopra, G., Weiss, A. \& Bluestone, J.A. CD28 costimulation: from mechanism to therapy. Immunity 44, 973-988 (2016).

84. Qureshi, O.S. et al. Trans-endocytosis of CD80 and CD86: a molecular basis for the cell-extrinsic function of CTLA-4. Science 332, 600-603 (2011).

85. Scalapino, K.J. \& Daikh, D.I. CTLA-4: a key regulatory point in the control of autoimmune disease. Immunol. Rev. 223, 143-155 (2008).

86. Travis, M.A. \& Sheppard, D. TGF-beta activation and function in immunity. Annu. Rev. Immunol. 32, 51-82 (2014).

87. Li, M.O., Wan, Y.Y. \& Flavell, R.A. T cell-produced transforming growth factor-beta1 controls T cell tolerance and regulates Th1- and Th17-cell differentiation. Immunity 26, 579-591 (2007).

88. Gutcher, I., Donkor, M.K., Ma, Q., Rudensky, A.Y., Flavell, R.A. \& Li, M.O. Autocrine transforming growth factor-beta1 promotes in vivo Th17 cell differentiation. Immunity 34, 396-408 (2011).

89. Franke, A. et al. Sequence variants in IL10, ARPC2 and multiple other loci contribute to ulcerative colitis susceptibility. Nat. Genet. 40, 1319-1323 (2008).

90. Engelhardt, K.R. et al. Clinical outcome in IL-10- and IL-10 receptordeficient patients with or without hematopoietic stem cell transplantation. J. Allergy Clin. Immunol. 131, 825-830 (2013).

91. Kuhn, R., Lohler, J., Rennick, D., Rajewsky, K. \& Muller, W. Interleukin-10deficient mice develop chronic enterocolitis. Cell 75, 263-274 (1993).

92. Roers, A. et al. Tcell-specific inactivation of the interleukin 10 gene in mice results in enhanced $T$ cell responses but normal innate responses to lipopolysaccharide or skin irritation. J. Exp. Med. 200, 1289-1297 (2004).

93. Rubtsov, Y.P. et al. Regulatory $T$ cell-derived interleukin-10 limits inflammation at environmental interfaces. Immunity 28, 546-558 (2008).

94. Maynard, C.L. et al. Regulatory T cells expressing interleukin 10 develop from Foxp3(+) and Foxp3( - ) precursor cells in the absence of interleukin 10. Nat. Immunol. 8, 931-941 (2007).

95. Liu, Z., Gerner, M.Y., Van Panhuys, N., Levine, A.G., Rudensky, A.Y. \& Germain, R.N. Immune homeostasis enforced by co-localized effector and regulatory T cells. Nature 528, 225-230 (2015).

96. Macpherson, A.J., McCoy, K.D., Johansen, F.E. \& Brandtzaeg, P. The immune geography of IgA induction and function. Mucosal Immunol. 1, 11-22 (2008).

97. Pabst, O. New concepts in the generation and functions of IgA. Nat. Rev. Immunol. 12, 821-832 (2012).

98. Fagarasan, S., Kawamoto, S., Kanagawa, O. \& Suzuki, K. Adaptive immune regulation in the gut: Tcell-dependent and Tcell-independent IgA synthesis. Annu. Rev. Immunol. 28, 243-273 (2010).

99. Macpherson, A.J., Gatto, D., Sainsbury, E., Harriman, G.R., Hengartner, H. \& Zinkernagel, R.M. A primitive T cell-independent mechanism of intestinal mucosal IgA responses to commensal bacteria. Science $\mathbf{2 8 8}$, 2222-2226 (2000).

100. Bemark, M., Boysen, P. \& Lycke, N.Y. Induction of gut IgA production through T cell-dependent and T cell-independent pathways. Ann. N. Y. Acad. Sci. 1247, 97-116 (2012).

101. Lindner, C. et al. Age, microbiota, and T cells shape diverse individual IgA repertoires in the intestine. J. Exp. Med. 209, 365-377 (2012).
102. Bunker, J.J. et al. Innate and adaptive humoral responses coat distinct commensal bacteria with immunoglobulin A. Immunity 43, 541-553 (2015).

103. Palm, N.W. et al. Immunoglobulin A coating identifies colitogenic bacteria in inflammatory bowel disease. Cell 158, 1000-1010 (2014).

104. Tang, C. et al. Inhibition of dectin-1 Signaling ameliorates colitis by inducing lactobacillus-mediated regulatory $\mathrm{T}$ cell expansion in the intestine. Cell Host Microbe 18, 183-197 (2015).

105. Kullberg, M.C. et al. Helicobacter hepaticus triggers colitis in specificpathogen-free interleukin-10 (IL-10)-deficient mice through an IL-12and gamma interferon-dependent mechanism. Infect. Immun. 66, 5157-5166 (1998).

106. Stepankova, R. et al. Segmented filamentous bacteria in a defined bacterial cocktail induce intestinal inflammation in SCID mice reconstituted with CD45RBhigh CD4 + T cells. Inflamm. Bowel Dis. 13, 1202-1211 (2007).

107. Ivanov, II et al. Induction of intestinal Th17 cells by segmented filamentous bacteria. Cell 139, 485-498 (2009).

108. Robertson, B.R. et al. Mucispirillum schaedleri gen. nov., sp. nov., a spiralshaped bacterium colonizing the mucus layer of the gastrointestinal tract of laboratory rodents. Int. J. Syst. Evol. Microbiol. 55 (Pt 3), 1199-1204 (2005).

109. Kau, A.L. et al. Functional characterization of IgA-targeted bacterial taxa from undernourished Malawian children that produce diet-dependent enteropathy. Sci. Transl. Med. 7, 276ra-224 (2015).

110. Hirota, K. et al. Plasticity of Th17 cells in Peyer's patches is responsible for the induction of T cell-dependent IgA responses. Nat. Immunol. 14, 372-379 (2013).

111. Cong, Y., Feng, T., Fujihashi, K., Schoeb, T.R. \& Elson, C.O. A dominant, coordinated T regulatory cell-IgA response to the intestinal microbiota. Proc. Natl Acad. Sci. USA 106, 19256-19261 (2009).

112. Karimi, K., Inman, M.D., Bienenstock, J. \& Forsythe, P. Lactobacillus reuteri-induced regulatory $T$ cells protect against an allergic airway response in mice. Am. J. Respir. Crit. Care Med. 179, 186-193 (2009).

113. Peterson, D.A., McNulty, N.P., Guruge, J.L. \& Gordon, J.I. IgA response to symbiotic bacteria as a mediator of gut homeostasis. Cell Host Microbe 2, 328-339 (2007)

114. Kawamoto, S. et al. Foxp3(+) Tcells regulate immunoglobulin a selection and facilitate diversification of bacterial species responsible for immune homeostasis. Immunity 41, 152-165 (2014).

115. Tsuji, M. et al. Preferential generation of follicular $B$ helper $T$ cells from Foxp3 + Tcells in gut Peyer's patches. Science 323, 1488-1492 (2009).

116. Komatsu, N., Mariotti-Ferrandiz, M.E., Wang, Y., Malissen, B., Waldmann, H. \& Hori, S. Heterogeneity of natural Foxp3 + Tcells: a committed regulatory T-cell lineage and an uncommitted minor population retaining plasticity. Proc. Natl Acad. Sci. USA 106, 1903-1908 (2009).

117. Chung, $Y$. et al. Follicular regulatory $T$ cells expressing Foxp3 and Bcl-6 suppress germinal center reactions. Nat. Med. 17, 983-988 (2011).

118. Linterman, M.A. et al. Foxp3 + follicular regulatory T cells control the germinal center response. Nat. Med. 17, 975-982 (2011).

119. Wollenberg, I. et al. Regulation of the germinal center reaction by Foxp3 + follicular regulatory T cells. J. Immunol. 187, 4553-4560 (2011).

120. Thorstenson, K.M. \& Khoruts, A. Generation of anergic and potentially immunoregulatory CD25 + CD4 Tcells in vivo after induction of peripheral tolerance with intravenous or oral antigen. J. Immunol. 167, 188-195 (2001).

121. Zhang, X., Izikson, L., Liu, L. \& Weiner, H.L. Activation of CD25(+) $\mathrm{CD} 4(+)$ regulatory Tcells by oral antigen administration. J. Immunol. 167, 4245-4253 (2001).

122. Kim, K.S. et al. Dietary antigens limit mucosal immunity by inducing regulatory T cells in the small intestine. Science 351, 858-863 (2016).

123. Hickey, C.A. et al. Colitogenic bacteroides thetaiotaomicron antigens access host immune cells in a sulfatase-dependent manner via outer membrane vesicles. Cell Host Microbe 17, 672-680 (2015).

124. Veenbergen, S. et al. Colonic tolerance develops in the iliac lymph nodes and can be established independent of CD103 dendritic cells. Mucosal Immunol. 9, 894-906 (2015).

125. Shale, M., Schiering, C. \& Powrie, F. CD4(+) T-cell subsets in intestinal inflammation. Immunol. Rev. 252, 164-182 (2013). 
126. Kieper, W.C. et al. Recent immune status determines the source of antigens that drive homeostatic Tcell expansion. J. Immunol. 174, 3158-3163 (2005).

127. Kim, J.M., Rasmussen, J.P. \& Rudensky, A.Y. Regulatory T cells prevent catastrophic autoimmunity throughout the lifespan of mice. Nat. Immunol. 8, 191-197 (2007).

128. Belkaid, Y., Piccirillo, C.A., Mendez, S., Shevach, E.M. \& Sacks, D.L. CD4 + CD25 + regulatory T cells control Leishmania major persistence and immunity. Nature 420, 502-507 (2002).
129. Hill, J.A. et al. Retinoic acid enhances Foxp3 induction indirectly by relieving inhibition from CD4 + CD44hi Cells. Immunity 29, 758-770 (2008).

130. Worbs, T., Hammerschmidt, S.I. \& Forster, R. Dendritic cell migration in health and disease. Nat. Rev. Immunol. 17, 30-48 (2017).

131. Knoop, K.A., Miller, M.J. \& Newberry, R.D. Transepithelial antigen delivery in the small intestine: different paths, different outcomes. Curr. Opin. Gastroenterol. 29, 112-118 (2013). 